\title{
Fujifilm SILVAMP TB LAM assay on cerebrospinal fluid for the detection of tuberculous meningitis in HIV-infected adults
}

Carson M Quinn ${ }^{1,2}$, Enock Kagimu², Michael Okirworth ${ }^{2}$, Ananta S Bangdiwala ${ }^{3}$, Gerald Mugumya $^{4}$, Prashanth S Ramachandran ${ }^{5}$, Michael R Wilson ${ }^{5}$, David B Meya ${ }^{2}$, Fiona V Cresswell $^{6}$, Nathan C Bahr ${ }^{\star 7}$, David R Boulware ${ }^{\star 8}$

1: School of Medicine, University of California, San Francisco, USA

2: Infectious Diseases Institute, College of Health Sciences, Makerere University, Kampala, Uganda

3: Department of Biostatistics, University of Minnesota, Minneapolis, USA

4: Microbiology Laboratory, Kiruddu National Referral Hospital, Kampala, Uganda

5: Weill Institute for Neurosciences, Department of Neurology, University of California, San Francisco, USA

6: Clinical Research Division, London School of Hygiene and Tropical Medicine, London, UK

7: Division of Infectious Diseases, Department of Medicine, University of Kansas, Kansas City, USA

8: Division of Infectious Diseases, Department of Internal Medicine, University of Minnesota, Minneapolis, USA

*: authors contributed equally 
Corresponding author: Carson M Quinn: School of Medicine, University of California, San Francisco, San Francisco, USA, 94143 carson.quinn@ucsf.edu

Summary of main point: The Fujifilm SILVAMP TB LAM assay on cerebrospinal fluid performs better than the prior LAM assay at detection of tuberculous meningitis in a Ugandan, predominantly HIV-positive cohort. 


\section{Abstract}

Background: Tuberculous meningitis (TBM) has a high fatality rate with inadequate diagnostic tests being a major contributor. The rollout of Xpert MTB/Rif and Xpert MTB/Rif Ultra (Xpert Ultra) have improved time-to-diagnosis with sensitivities similar to culture, yet test availability and sensitivity are inadequate. The TB lipoarabinomannan lateral flow assay (AlereLAM) offers ease of use, but its low sensitivity in cerebrospinal fluid (CSF) limits clinical utility for TBM. The Fujifilm SILVAMP TB LAM (FujiLAM) assay has excellent sensitivity in urine, but performance on cerebrospinal fluid is uncertain.

Methods: We conducted a prospective cohort study at Kiruddu National Referral Hospital in Kampala, Uganda enrolling patients suspected to have TB meningitis. CSF was tested using AlereLAM, Xpert Ultra, culture, and FujiLAM. Results were compared to two reference standards: probable and definite TBM, or definite TBM alone by the uniform TBM case definition.

Results: Of 101 patients enrolled (95/101 HIV-positive), 34 had definite TBM and 24 had probable TBM. FujiLAM sensitivity on CSF was 52\% (30/58) for definite or probable TBM, compared to 55\% (32/58) for Xpert Ultra. AlereLAM had lower sensitivity than FujiLAM in the subgroup of patients tested with both assays ( $14 \%, 4 / 28$ vs. $50 \%, 14 / 28 ; p<0.01)$. FujiLAM specificity was $98 \%$ (42/43) for patients without probable or definite TBM.

Conclusions: FujiLAM showed higher sensitivity than AlereLAM, with sensitivity potentially approaching that of Xpert Ultra. FujiLAM could improve time-to-treatment-initiation, especially in settings where the more technical Xpert Ultra system might not be feasible. Large confirmatory studies are needed.

Key words: Mycobacterium tuberculosis, Tuberculous meningitis, Lipoarabinomannan, Diagnostic Accuracy, Fujifilm SILVAMP TB LAM 


\section{Introduction}

Tuberculosis is a major cause of death globally, and tuberculous meningitis (TBM) is its most severe complication, with a case-fatality rate estimated at $50-60 \%$ in people living with HIV (PLHIV) [1]. Late initiation of TBM treatment due to limited diagnostic options, especially in low-resource settings, contributes to high mortality [2,3]. The traditional gold standard, mycobacterial culture of cerebrospinal fluid (CSF), is limited by slow time-topositivity that makes it unhelpful for diagnosis in this rapidly progressing disease [4]. Microscopy smear for acid-fast bacilli, while rapid, is highly insensitive in most settings [5]. The GeneXpert MTB/RIF Ultra cartridge-based PCR assay (Xpert Ultra) improved on the 50 to $60 \%$ sensitivity of its predecessor (GeneXpert MTB/Rif, Xpert) to reported sensitivities of $70-77 \%$ for diagnosis of TBM $[4,6,7]$. However, barriers to implementation of Xpert Ultra, including reliable electricity, cost, initial acquisition of the platform, and turnaround time when there may be a queue of samples awaiting processing might exist in the low-resource settings with especially high TBM burden [8-10].

Detection of the mycobacterial cell wall component lipoarabinomannan (LAM) in urine has proven useful for the detection of disseminated TB in immunosuppressed patients [11]. The Alere Determine TB LAM (AlereLAM, Abbott, Chicago, IL, USA) results with a turnaround-time of 25 minutes, and requires minimal training and no additional materials, making it ideal for use in resource-constrained settings. The Fujifilm SILVAMP TB LAM assay (FujiLAM, FujiFilm, Tokyo, Japan), which targets novel LAM epitopes and includes a silver amplification step [12], has been used on urine to diagnose TB in PLHIV with higher sensitivity than AlereLAM $[13,14]$. A recent meta-analysis of 1,595 HIV-positive adults demonstrated $70.7 \%$ sensitivity of urine FujiLAM in diagnosing microbiologically-confirmed pulmonary and extrapulmonary TB compared to $34.9 \%$ sensitivity with urine AlereLAM; the reference standard consisted of PCR, culture, or smear positivity of urine, blood, or sputum [15]. An initial proof-of-concept study using AlereLAM on CSF acquired post-mortem to detect TBM showed encouraging results compared to autopsy histopathologic TBM 
diagnosis with $75 \%$ sensitivity [16]; however, subsequent studies using clinical CSF samples showed low sensitivity (0-33\%) of AlereLAM in diagnosing TBM, suggesting limited clinical utility [17-19]. A strong relationship has been established between LAM detection and low CD4 count [20], which was replicated using the FujiLAM assay in urine [15], and seems to apply to TBM diagnosis using a CSF LAM assay [21]. One study has evaluated the sensitivity of urine FujiLAM in diagnosing TBM in a small cohort $(n=19)$ of microbiologically confirmed cases (47\% sensitivity) [22]; however, performance of FujiLAM in CSF is unclear. We compared the diagnostic performance of FujiLAM, AlereLAM, culture, and Xpert Ultra on 101 prospectively collected CSF samples of patients with clinician-suspected possible TBM.

\section{Methods}

We conducted a prospective cohort study screening consecutive adults with suspected meningitis (i.e. presentation with some combination of headache, fever, nuchal rigidity, neurologic deficit, or altered mental status) at Kiruddu National Referral Hospital in Kampala, Uganda, nested within the screening process of an observational meningitis diagnostic trial and a TB meningitis randomized controlled trial (ISRCTN42218549). All participants or their surrogates provided written informed consent. Institutional review board approvals were obtained from the Mulago Research and Ethics Committee, Uganda National Council of Science and Technology, and the University of Minnesota.

After lumbar puncture, physicians performed bedside cryptococcal antigen lateral flow assay (CrAg; IMMY, Norman, OK, USA) testing, alongside point-of-care assays for glucose and lactate. Participants without cryptococcosis were then evaluated for TB and bacterial meningitis [23]. We enrolled those patients with adequate CSF for FujiLAM and Xpert Ultra testing, and clinician-suspected TB meningitis after initial lumbar puncture, based on negative CSF CrAg ruling out cryptococcal meningitis, the most common etiology of meningitis in our setting [9]. After bedside testing, $1 \mathrm{~mL}$ of CSF was used for routine testing (white cell count, protein, Gram stain, Ziehl-Neelsen stain), 1-2mL was used for 
mycobacterial culture, $2 \mathrm{~mL}$ was used for Xpert Ultra, and $0.2 \mathrm{~mL}$ for Alere and FujiLAM testing. Fresh samples were tested within two hours of collection. The remainder was cryopreserved at $-80^{\circ} \mathrm{C}$ for future testing. When there was limited CSF volume, AlereLAM and culture were not always performed; culture was prioritized, except when there was supply disruption. Repeat lumbar punctures were performed at the clinician's discretion, and when possible, Xpert Ultra was repeated in cases where it was initially negative if clinical suspicion for TBM remained high. All tests were performed on whole CSF without prior processing or concentration. CSF culture used mycobacteria growth indicator tube (MGIT) with a Bactec960 instrument (Becton Dickinson, Franklin Lakes, NJ, USA). We performed Xpert Ultra according to a published protocol [6]. AlereLAM was run on two drops of CSF; results were read by a trained laboratory staff member, blinded to other TB diagnostic results, in consultation with the test's reference scale card. All assays were run prospectively in parallel, except FujiLAM for those subjects screened before the assay was available, or during periods of disruption of the supply of tests. For those subjects, FujiLAM was run on cryopreserved specimens. The FujiLAM assay was performed according to manufacturer instructions and was read independently by two readers who were blinded to other CSF TB diagnostic testing. In case of discordance, the strip was read by a third reader, and the teststrip was re-inspected for mutual agreement on a final result. As is outlined in the FujiLAM assay instructions, any line identified on the test line was deemed positive, no matter how faint. Invalid results, defined by the manufacturer as a lack of control line, were repeated when adequate CSF remained, with this repeat result counting towards the final analysis, but otherwise were counted as negative for purposes of statistical analysis.

We assessed test diagnostic performance against two reference standards, both based upon the consensus uniform case definition for TBM [24]. Definite TBM was defined by detection of Mycobacterium tuberculosis (MTB) on a molecular or microbiological assay: Xpert Ultra (including when repeated on a subsequent LP from the same hospitalization), MGIT culture, or Ziehl-Neelsen stain. We defined the second reference standard as definite 
TBM or probable TBM defined as $\geq 10$ points on the diagnostic scoring system when brain imaging is not available or $\geq 12$ points when brain imaging is available; this score was evaluated for all included participants by an expert reviewer [24]. The case definition includes ancillary TB diagnostics, which were performed based on patient symptoms, and included sputum Xpert Ultra, urine AlereLAM and Xpert Ultra, chest radiography, and abdominal ultrasound. Of note, only the Xpert Ultra result from the initial LP counted towards the diagnostic performance of Xpert Ultra (e.g. repeat testing was not counted in its favor, but did contribute to the reference standard). Xpert Ultra provides semi-quantitative results of "trace", "very low", "low", and "medium", thought to correlate with bacillary load and time-toculture-positivity [6]. All categories were counted as positive.

We calculated sensitivity, specificity, and positive predictive value against the reference standards. We compared baseline clinical characteristics and demographic data by diagnosis and separately by FujiLAM result using Mann-Whitney $U$ for continuous variables and Fisher's exact test for categorical variables. We evaluated concordance between diagnostic assays with McNemar's test. We evaluated the trend in FujiLAM sensitivity across Xpert Ultra semi-quantitative categories using the Cochrane-Armitage test for trend. Diagnostic performance of MGIT culture and AlereLAM was evaluated in secondary analyses which were limited to those patients whose CSF was run on all relevant assays. All analyses were conducted using STATA version 15.

\section{Results}

Of 587 patients presenting with meningitis between May 18, 2018, and March 9, 2020, 101 were suspected to have TBM, and had CSF tested on Xpert Ultra and FujiLAM (Figure 1). FujiLAM was tested on fresh samples for 22 patients, and cryopreserved samples for 79 patients. Of these 101 patients, 48 also had CSF MGIT cultures performed and 36 had CSF AlereLAM testing performed. We diagnosed definite TBM in 34 patients (34\%) based on the microbiologic reference standard (Xpert Ultra $n=32$, MTB culture $n=15$ ). 
Probable TBM was diagnosed in an additional 24 patients, for a total of 58 patients with definite or probable TBM. Of the remaining patients, 27 were classified by the uniform case definition as possible TBM, and 16 were not TBM. 95 patients were HIV-positive, 6 were HIV-negative (including 1 definite TBM case). Baseline demographic and clinical data are listed in Table 1 based on probable/definite TBM diagnosis, and FujiLAM positivity. The FujiLAM positive group was younger and had more inflammatory CSF, as did the group diagnosed with TBM. Patients with positive FujiLAM were more likely to have a low baseline CD4 T cell count, which was not observed in patients diagnosed with TBM.

FujiLAM sensitivity was $74 \%(25 / 34 ; 95 \% \mathrm{Cl}, 56 \%-87 \%)$ versus definite TBM, as opposed to $94 \%(32 / 34 ; 95 \% \mathrm{Cl}, 80 \%-99 \%)$ for Xpert Ultra (Figure 2a). Against this reference standard, sensitivity of Xpert Ultra was significantly higher than FujiLAM ( $p=0.02)$. The one false negative by both FujiLAM and initial Xpert Ultra was positive by culture (21 days to positivity) and repeat day 14 Xpert Ultra. FujiLAM was positive on the CSF from 6 of 67 patients without definite TBM (specificity = 91\%; $95 \% \mathrm{Cl}, 82 \%-97 \%$ ). Positive predictive value was $81 \%(25 / 31 ; 95 \% \mathrm{Cl}, 63 \%-93 \%)$ if those were false positives.

When compared to the reference standard of definite or probable TBM, FujiLAM had a sensitivity of $52 \%(30 / 58 ; 95 \% \mathrm{Cl}, 38-65)$, compared to $55 \%(32 / 58 ; 95 \% \mathrm{Cl}, 42 \%-68 \%)$ for Xpert Ultra (Figure 2b). The difference in sensitivity displayed by FujiLAM and Xpert Ultra was not statistically significant $(p=0.6)$. Used together, FujiLAM and Xpert Ultra have a $66 \%$ (38/58; $95 \% \mathrm{Cl}, 52 \%-77 \%)$ sensitivity for definite or probable TBM, providing an increased sensitivity of $11 \%$ over Xpert Ultra alone, with potential decreased specificity. FujiLAM specificity was $98 \%(42 / 43 ; 95 \% \mathrm{Cl} 87 \%-100 \%)$, with the false positive in a patient with culture-confirmed non-tuberculous mycobacterial meningitis. FujiLAM was positive in 5 patients with probable TBM, all of which had evidence of disseminated TB, and 3 of which died within 90 days. MGIT culture was performed in 4/5 cases; all were negative. These 5 cases had less inflammatory CSF than the TBM cohort as a whole (4/5 with acellular CSF). 
Sensitivity (51\% and 52\%) and specificity (100\% and 97\%) did not differ between fresh $(\mathrm{n}=23)$ and cryopreserved $(\mathrm{n}=78)$ samples respectively.

As there was inadequate CSF or supplies to perform AlereLAM and culture on all samples, we undertook secondary analyses of only those patients with complete results to allow for direct comparison of AlereLAM and culture sensitivity to FujiLAM. In the subgroup of patients with AlereLAM, FujiLAM, and Xpert Ultra results $(n=36)$, FujiLAM had a sensitivity of $50 \%(14 / 28 ; 95 \% \mathrm{Cl}, 31 \%-69 \%)$ and AlereLAM had a sensitivity of $14 \%(4 / 28 ; 95 \% \mathrm{Cl}$, $4 \%-33 \%$, versus the probable or definite TBM reference standard. Compared to definite TBM, FujiLAM had a sensitivity of $65 \%(11 / 17 ; 95 \% \mathrm{Cl}, 38 \%-86 \%)$ and AlereLAM had a sensitivity of $24 \%(4 / 17 ; 95 \% \mathrm{Cl}, 7 \%-50 \%)$. Sensitivity of FujiLAM was significantly improved from AlereLAM against both reference standards ( $p<0.01$ for probable/definite; $p<0.01$ for definite).

In the subgroup of patients with culture, FujiLAM, and Xpert Ultra results ( $n=48)$, FujiLAM had a sensitivity of $63 \%(19 / 30 ; 95 \% \mathrm{Cl}, 44 \%-80 \%)$ and culture had a sensitivity of $50 \%(15 / 30,95 \% \mathrm{Cl}, 31 \%-69 \%)$, versus the definite or probable TBM reference standard (Figure 3). Compared to definite TBM, FujiLAM had a sensitivity of 79\% (15/19; $95 \% \mathrm{Cl}$, $54 \%-94 \%)$ and culture had a sensitivity of $79 \%(15 / 19 ; 95 \% \mathrm{Cl}, 54 \%-94 \%)$. The difference between the sensitivity of culture and FujiLAM against either reference standard did not meet statistical significance ( $p=0.25$ for probable/definite; $p=1$ for definite).

To determine whether FujiLAM sensitivity was related to TB bacillary load, we calculated sensitivity in Xpert Ultra-positive patients sub-grouped by semi-quantitative Xpert Ultra category. In patients with the lowest PCR burden ("trace"), FujiLAM sensitivity was 58\% $(7 / 12,95 \% \mathrm{Cl}, 28 \%-85 \%)$. In the intermediate category ("very low"), FujiLAM sensitivity was $75 \%(6 / 8 ; 95 \% \mathrm{Cl}, 35 \%-97 \%)$. In the highest PCR burden patients ("low" and "medium" combined), FujiLAM sensitivity was $92 \%(11 / 12,95 \% \mathrm{Cl}, 62 \%-100 \%)$. In patients who were negative on Xpert Ultra, but positive by culture or categorized as probable TBM by the case definition, FujiLAM sensitivity was $23 \%(6 / 26,95 \% \mathrm{Cl}, 9 \%-44 \%)$. This demonstrates a 
significant increasing trend in FujiLAM sensitivity across increasing Xpert Ultra semiquantitative categories $(p<0.0001)$.

FujiLAM test did not return a valid result in 3 of 101 patients; 1 had adequate remaining sample to be repeated and was positive, counting towards the final analysis. Cryopreserved samples were stored at $-80^{\circ} \mathrm{C}$ for a median of 424 days (IQR 229-579) before being thawed and tested. Inter-reader agreement of FujiLAM results was $94 \%$ with Kappa $0.86(95 \% \mathrm{Cl} 0.67-1$, SE 0.099$)$; a majority of initial disagreement was due to very faint result lines that required further inspection for one reader to visualize.

\section{Discussion}

In this cohort of predominantly HIV-positive patients with clinical suspicion of TBM, the new Fujifilm LAM system detected TB in the CSF of as many TBM cases as culture and nearly as many as Xpert Ultra. FujiLAM had more than double the sensitivity of the previously available LAM lateral flow assay.

While FujiLAM was falsely negative for some patients with positive Xpert Ultra tests, these data suggest that FujiLAM tends to be positive in the highest risk groups of TBM patients. Sensitivity is highest in those with the greatest Xpert Ultra-determined bacillary load, a group previously shown to have increased mortality [25]. FujiLAM-positive patients are more likely to be profoundly immunosuppressed, and tend to have higher mortality, than those who are negative. FujiLAM therefore could serve as a rapid diagnostic to ensure quick treatment initiation in patients who stand to benefit most.

Several patients met the criteria for probable TBM whose CSF was negative on Ultra, but positive on FujiLAM. It has previously been noted in urine that LAM-based and PCRbased assays detect different subsets of TB patients, although the mechanism is not clear [26]. One explanation for our findings in CSF is that a population of TBM patients can be detected by FujiLAM but not Xpert Ultra, perhaps due to CSF presence of cell-wall antigens 
without abundant intact bacilli or nucleic acid detectable by Xpert Ultra. This difference could also simply be due to chance and the presence of TB in the tested CSF sample in a patient with low bacillary load. Alternately, since many probable TBM cases have confirmed disseminated TB, FujiLAM might detect LAM antigen from an extra-meningeal MTB source that passed through a non-intact blood-brain barrier into otherwise MTB-free CSF, where it elicits an immune response. A final possibility is undetected non-TB mycobacterial infection of the central nervous system, which our findings suggest can lead to FujiLAM positivity, and has been shown to cause false positives on urine FujiLAM [15]. However, the concordance between FujiLAM positivity and probable TBM classification, as well as the high mortality in this group, suggests that these are true positives and should be treated as such. Given uncertainty concerning optimal treatment of HIV-associated TBM, the clinical impact of FujiLAM positivity in a probable TBM patient with otherwise negative CSF diagnostics is unclear. Anti-tubercular therapy, already indicated in patients diagnosed with disseminated TB, does not differ between TBM and extra-meningeal TB, although this may change based on ongoing trials of intensified therapy [27]. However, TBM treatment also includes adjunctive corticosteroids, which have the potential to harm patients with disseminated TB without TBM. In fact, whether corticosteroids are beneficial in PLIV remains unclear and is the subject of ongoing study [28]. In our setting, empiric corticosteroids in cases of disseminated TB meeting criteria of probable TBM are added by clinician judgement.

Given moderate reported sensitivity (47\%) of urine FujiLAM in detecting confirmed TBM, there is potential for improving diagnostic yield by testing both urine and CSF [22]. However, without a robust study investigating both sample types simultaneously, it is difficult to speculate whether dual-specimen FujiLAM testing would improve diagnostic performance.

As this study was largely exploratory, we were limited by a 101 patient cohort, and therefore cannot show definitively if FujiLAM performs comparably to Xpert Ultra in TBM diagnosis. Another limitation was inconsistent inclusion of all possible TBM diagnostics, due to the availability of CSF volume or AlereLAM supplies. The protocol for Xpert Ultra used 
$2 \mathrm{~mL}$ of unconcentrated CSF rather than centrifuging $>5 \mathrm{~mL}$, a protocol that has been shown to be more sensitive for the original Xpert assay, possibly leading to underdiagnosis of definite TBM in this cohort [29]. Furthermore, the asymmetry in inclusion of Xpert Ultra in the definite TBM reference standard is inherently a source of incorporation bias, leading to the performance of existing tests to generally appear better, which could overestimate the true difference in sensitivity. This is suggested by the more similar sensitivity of Xpert Ultra and FujiLAM among those with definite or probable TBM. CSF mycobacterial culture was only performed on half the cohort, and AlereLAM on less, which limited our ability to compare these tests to FujiLAM. We also used both stored and fresh samples, which could, despite similarity in the results between these groups, limit generalizability of the findings to point-ofcare usage. In our setting we have seen similar performance of Xpert Ultra using cryopreserved or fresh CSF samples [4,6]. Inter-reader agreement on FujiLAM results was slightly lower in our study than in published results using urine $[14,15]$; this suggests potential for optimization of the testing protocol for CSF, and the importance of careful inspection of test results for the sometimes faint result lines occurring in CSF testing. These results, however, justify a larger study to test FujiLAM diagnostic performance for TBM in comparison Xpert Ultra and culture. As recent studies of Xpert Ultra from different sites with differing HIV prevalences have shown conflicting results [30], diagnostic validation studies are ideally conducted as multisite studies. Such a study could also better evaluate the incremental yield of adding FujiLAM in settings where Xpert Ultra is already available, as our results suggest that FujiLAM may detect some high-risk cases of TBM that Xpert Ultra does not. FujiLAM certainly shows promise for filling a niche in TBM diagnostics in settings where implementation of the more technical Xpert Ultra system might not be feasible or where the turnaround time of results is prolonged, warranting further study of this diagnostic modality. 


\section{NOTES}

Acknowledgements: We wish to thank study participants and the entire study team whose work is crucial to our ability to report these findings. We thank Dr. Kosuke Yasukawa for logistical support.

Funding: This work was supported by the National Institutes of Neurological Diseases and Stroke of the National Institutes of Health [K23NS110470 to N.C.B., K08NS096117 to M.R.W.

and R01NS086312 to D.R.B.]. M.R.W. is also supported by the National Institute of Allergy and Infectious Diseases [R01Al145437]. F.V.C. is supported by a Wellcome Clinical PhD Fellowship [210772/Z/18/Z].

Conflict of Interest: FujiFilm provided the SILVAMP TB LAM tests for this study. MRW reports grants form Roche/Genentech, outside the submitted work. No authors have any financial conflict of interest. 


\section{References}

1. Woldeamanuel YW, Girma B. A 43-year systematic review and meta-analysis: casefatality and risk of death among adults with tuberculous meningitis in Africa. J Neurol 2014; 261:851-865.

2. Sheu J-J, Yuan R-Y, Yang C-C. Predictors for outcome and treatment delay in patients with tuberculous meningitis. Am J Med Sci 2009; 338:134-139.

3. Bahr NC, Boulware DR. Methods of rapid diagnosis for the etiology of meningitis in adults. Biomark Med 2014; 8:1085-1103.

4. Bahr NC, Nuwagira E, Evans EE, et al. Diagnostic accuracy of Xpert MTB/RIF Ultra for tuberculous meningitis in HIV-infected adults: a prospective cohort study. Lancet Infect Dis 2018; 18:68-75.

5. Heemskerk AD, Donovan J, Thu DDA, et al. Improving the microbiological diagnosis of tuberculous meningitis: A prospective, international, multicentre comparison of conventional and modified Ziehl-Neelsen stain, GeneXpert, and culture of cerebrospinal fluid. J Infect 2018; 77:509-515.

6. Cresswell FV, Tugume L, Bahr NC, et al. Xpert MTB/RIF Ultra for the diagnosis of HIV-associated tuberculous meningitis: a prospective validation study. Lancet Infect Dis 2020; 20:308-317.

7. Donovan J, Thu DDA, Phu NH, et al. Xpert MTB/RIF Ultra versus Xpert MTB/RIF for the diagnosis of tuberculous meningitis: a prospective, randomised, diagnostic accuracy study. Lancet Infect Dis 2020; 20:299-307.

8. Jarvis JN, Meintjes G, Williams A, Brown Y, Crede T, Harrison TS. Adult meningitis in a setting of high HIV and TB prevalence: findings from 4961 suspected cases. BMC Infect Dis 2010; 10:67.

9. Ellis J, Bangdiwala AS, Cresswell FV, et al. The Changing Epidemiology of HIVAssociated Adult Meningitis, Uganda 2015-2017. Open Forum Infect Dis 2019; 6:ofz419.

10. Cazabon D, Alsdurf H, Satyanarayana S, et al. Quality of tuberculosis care in high burden countries: the urgent need to address gaps in the care cascade. Int $\mathrm{J}$ Infect Dis IJID Off Publ Int Soc Infect Dis 2017; 56:111-116.

11. Lawn SD, Kerkhoff AD, Burton R, et al. Diagnostic accuracy, incremental yield and prognostic value of Determine TB-LAM for routine diagnostic testing for tuberculosis in HIVinfected patients requiring acute hospital admission in South Africa: a prospective cohort. BMC Med 2017; 15:67.

12. Sigal GB, Pinter A, Lowary TL, et al. A Novel Sensitive Immunoassay Targeting the 5-Methylthio-d-Xylofuranose-Lipoarabinomannan Epitope Meets the WHO's Performance Target for Tuberculosis Diagnosis. J Clin Microbiol 2018; 56. 
13. Broger T, Sossen B, du Toit E, et al. Novel lipoarabinomannan point-of-care tuberculosis test for people with HIV: a diagnostic accuracy study. Lancet Infect Dis 2019; 19:852-861.

14. Bjerrum S, Broger T, Székely R, et al. Diagnostic Accuracy of a Novel and Rapid Lipoarabinomannan Test for Diagnosing Tuberculosis Among People With Human Immunodeficiency Virus. Open Forum Infect Dis 2019; 7:ofz530.

15. Broger T, Nicol MP, Székely R, et al. Diagnostic accuracy of a novel tuberculosis point-of-care urine lipoarabinomannan assay for people living with HIV: A meta-analysis of individual in- and outpatient data. PLoS Med 2020; 17:e1003113.

16. Cox JA, Lukande RL, Kalungi S, et al. Accuracy of Lipoarabinomannan and Xpert MTB/RIF Testing in Cerebrospinal Fluid To Diagnose Tuberculous Meningitis in an Autopsy Cohort of HIV-Infected Adults. J Clin Microbiol 2015; 53:2667-2673.

17. Siddiqi OK, Birbeck GL, Ghebremichael M, et al. Prospective Cohort Study on Performance of Cerebrospinal Fluid (CSF) Xpert MTB/RIF, CSF Lipoarabinomannan (LAM) Lateral Flow Assay (LFA), and Urine LAM LFA for Diagnosis of Tuberculous Meningitis in Zambia. J Clin Microbiol 2019; 57:e00652-19.

18. Kwizera R, Cresswell FV, Mugumya G, et al. Performance of Lipoarabinomannan Assay using Cerebrospinal fluid for the diagnosis of Tuberculous meningitis among HIV patients. Wellcome Open Res 2019; 4:123.

19. Bahr NC, Tugume L, Boulware DR. A Word of Caution in Considering the Use of the Lipoarabinomannan Lateral Flow Assay on Cerebrospinal Fluid for Detection of Tuberculous Meningitis. J Clin Microbiol 2016; 54:241-242.

20. Bjerrum S, Schiller I, Dendukuri N, et al. Lateral flow urine lipoarabinomannan assay for detecting active tuberculosis in people living with HIV. Cochrane Database Syst Rev 2019; 10:CD011420.

21. Patel VB, Singh R, Connolly C, et al. Comparison of a Clinical Prediction Rule and a LAM Antigen-Detection Assay for the Rapid Diagnosis of TBM in a High HIV Prevalence Setting. PLoS ONE 2010; 5:e15664.

22. Kerkhoff AD, Sossen B, Schutz C, et al. Diagnostic sensitivity of SILVAMP TB-LAM (FujiLAM) point-of-care urine assay for extra-pulmonary tuberculosis in people living with HIV. Eur Respir J 2020; 55:1901259.

23. Durski KN, Kuntz KM, Yasukawa K, Virnig BA, Meya DB, Boulware DR. Costeffective Diagnostic Checklists for Meningitis in Resource Limited Settings. J Acquir Immune Defic Syndr 1999 2013; 63:e101-e108.

24. Marais S, Thwaites G, Schoeman JF, et al. Tuberculous meningitis: a uniform case definition for use in clinical research. Lancet Infect Dis 2010; 10:803-812.

25. Martyn EM, Bangdiwala AS, Kagimu E, et al. Cerebrospinal Fluid Bacillary Load by Xpert MTB/RIF Ultra Polymerase Chain Reaction Cycle Threshold Value Predicts 2-Week Mortality in Human Immunodeficiency Virus-Associated Tuberculous Meningitis. Clin Infect 
Dis Available at: https://academic.oup.com/cid/advancearticle/doi/10.1093/cid/ciaa1444/5912549. Accessed 17 November 2020.

26. Cresswell FV, Ellis J, Kagimu E, et al. Standardized Urine-Based Tuberculosis (TB) Screening With TB-Lipoarabinomannan and Xpert MTB/RIF Ultra in Ugandan Adults With Advanced Human Immunodeficiency Virus Disease and Suspected Meningitis. Open Forum Infect Dis 2020; 7:ofaa100.

27. Seddon JA, Tugume L, Solomons R, Prasad K, Bahr NC, Tuberculous Meningitis International Research Consortium. The current global situation for tuberculous meningitis: epidemiology, diagnostics, treatment and outcomes. Wellcome Open Res 2019; 4:eCollection 2019.

28. Prasad K, Singh MB, Ryan H. Corticosteroids for managing tuberculous meningitis. Cochrane Database Syst Rev 2016; 4:CD002244.

29. Bahr NC, Tugume L, Rajasingham R, et al. Improved Diagnostic Sensitivity for TB Meningitis with Xpert MTB/Rif of centrifuged CSF: a prospective study. Int J Tuberc Lung Dis Off J Int Union Tuberc Lung Dis 2015; 19:1209-1215.

30. Davis AG, Wilkinson RJ. Diagnostic tests for tuberculous meningitis. Lancet Infect Dis 2020; 20:262-263. 
Table 1. Baseline Demographics by TBM diagnosis, and separately by result on the FujiLAM assay.

\begin{tabular}{|c|c|c|c|c|c|c|}
\hline Characteristic & $\begin{array}{c}\text { Definite or } \\
\text { Probable } \\
\text { TBM }\end{array}$ & Not TBM & $p$ value & $\begin{array}{l}\text { FujiLAM } \\
\text { Positive }\end{array}$ & $\begin{array}{l}\text { FujiLAM } \\
\text { Negative }\end{array}$ & $\begin{array}{c}\mathbf{p} \\
\text { value }\end{array}$ \\
\hline Age, years & $\begin{array}{c}33 \\
(26-40)\end{array}$ & $\begin{array}{c}37 \\
(29-48)\end{array}$ & 0.04 & $\begin{array}{c}32 \\
(26-40)\end{array}$ & $\begin{array}{c}37 \\
(28-45)\end{array}$ & 0.04 \\
\hline Women & $21(36 \%)$ & $16(37 \%)$ & 0.9 & $12(39 \%)$ & $25(36 \%)$ & 0.8 \\
\hline MRC grade 1 & $10(17 \%)$ & 15 (44\%) & 0.02 & $3(10 \%)$ & $22(36 \%)$ & 0.01 \\
\hline 2 & $35(60 \%)$ & 16 (47\%) & & $20(65 \%)$ & 31 (51\%) & \\
\hline 3 & $13(22 \%)$ & $3(9 \%)$ & & $8(26 \%)$ & $8(13 \%)$ & \\
\hline GCS less than 15 & $46(79 \%)$ & $23(55 \%)$ & 0.02 & $28(90 \%)$ & $41(59 \%)$ & 0.002 \\
\hline $\begin{array}{l}\text { Headache } \\
\text { symptom }\end{array}$ & $51(86 \%)$ & $32(76 \%)$ & 0.3 & 27 (87\%) & $55(80 \%)$ & 0.6 \\
\hline $\begin{array}{l}\text { Illness Duration, } \\
\text { days }\end{array}$ & $30(14-30)$ & $\begin{array}{c}21(14- \\
60)\end{array}$ & 0.39 & $\begin{array}{c}30(14- \\
60)\end{array}$ & $24.5(14-30)$ & 0.23 \\
\hline CD4 T cells/ $\mu \mathrm{L}$ & $\begin{array}{c}79 \\
(30-260)\end{array}$ & $\begin{array}{c}102 \\
(45-232)\end{array}$ & 0.48 & $\begin{array}{c}46 \\
(18-86)\end{array}$ & $\begin{array}{c}153 \\
(45-273)\end{array}$ & 0.04 \\
\hline$<100$ CD4 cells $/ \mu \mathrm{L}$ & 13 (57\%) & $4(44 \%)$ & 0.7 & $9(82 \%)$ & 8 (38\%) & 0.03 \\
\hline $\begin{array}{l}\text { Pulmonary or } \\
\text { extrapulmonary } \\
\text { TB }\end{array}$ & $27(47 \%)$ & $7(16 \%)$ & 0.002 & $15(48 \%)$ & $19(27 \%)$ & 0.04 \\
\hline
\end{tabular}




\begin{tabular}{|c|c|c|c|c|c|c|}
\hline $\begin{array}{l}\text { CSF WBC, } \\
\text { cells/ } \mu \mathrm{L}\end{array}$ & $\begin{array}{c}65 \\
(<5-245)\end{array}$ & $\begin{array}{c}<5 \\
(<5-35)\end{array}$ & 0.003 & $\begin{array}{c}87.5 \\
(<5-240)\end{array}$ & $\begin{array}{c}<5 \\
(<5-80)\end{array}$ & 0.03 \\
\hline $\begin{array}{l}\text { CSF protein, } \\
\mathrm{mg} / \mathrm{dL}\end{array}$ & $\begin{array}{c}116 \\
(72-184)\end{array}$ & $\begin{array}{c}74 \\
(36-118)\end{array}$ & 0.02 & $\begin{array}{c}119 \\
(72-187)\end{array}$ & $\begin{array}{c}99 \\
(53-134)\end{array}$ & 0.2 \\
\hline $\begin{array}{l}\text { CSF glucose, } \\
\mathrm{mg} / \mathrm{dL}\end{array}$ & $\begin{array}{c}29 \\
(19-68)\end{array}$ & $\begin{array}{c}82 \\
(65-107)\end{array}$ & $<0.001$ & $\begin{array}{c}22 \\
(18-54)\end{array}$ & $\begin{array}{c}75 \\
(36-100)\end{array}$ & 0.005 \\
\hline $\begin{array}{l}\text { CSF lactate, } \\
\mathrm{mmol} / \mathrm{L}\end{array}$ & $\begin{array}{c}7.5 \\
(3.9-9.5)\end{array}$ & $\begin{array}{c}2.3 \\
(2.0-3.1)\end{array}$ & $<0.001$ & $\begin{array}{c}9.5 \\
(4.3-10)\end{array}$ & $\begin{array}{c}2.9 \\
(2-7.2)\end{array}$ & 0.002 \\
\hline 14 day mortality & 17 (29\%) & $9(21 \%)$ & 0.5 & $10(32 \%)$ & $16(23 \%)$ & 0.3 \\
\hline
\end{tabular}

Values are $\mathrm{N}(\%)$ or medians with interquartile ranges (IQR). GCS= Glasgow coma scale, $M R C=$ British medical research council grade for TBM (This grade was available for $n=92$ participants). CD4 T cell count was only available for the latter portion of the cohort $(n=32)$. Pulmonary or extrapulmonary TB is based upon detection by Xpert Ultra or AlereLAM in sputum or urine, or suggestive chest radiography or abdominal ultrasound. 


\section{FIGURE LEGENDS}

Figure 1. Consort Diagram for inclusion in study. Probable or definite Tuberculous Meningitis (TBM) are defined according to the uniform case definition. FujiLAM=Fujifilm SILVAMP TB LAM

Figure 2. Venn Diagram of overlap of FujiLAM and Xpert Ultra in diagnosing TB Meningitis in the full cohort $(n=101)$. Included are true positives based on the reference standards of (a) Definite TBM and (b) Probable or Definite TBM. There is significant overlap, but neither assay displayed perfect sensitivity. FujiLAM= FujiFilm SILVAMP TB LAM assay, TBM= Tuberculous meningitis

Figure 3. Venn diagram of overlap in tuberculous meningitis diagnostics in 48 patients for whom there were results for FujiLAM, Xpert Ultra, and MGIT Culture. Included are true positives based on a reference standard of probable or definite TBM. FujiLAM= FujiFilm SILVAMP TB LAM assay, TBM= Tuberculous meningitis, MGIT = Mycobacteria Growth Indicator Tube 


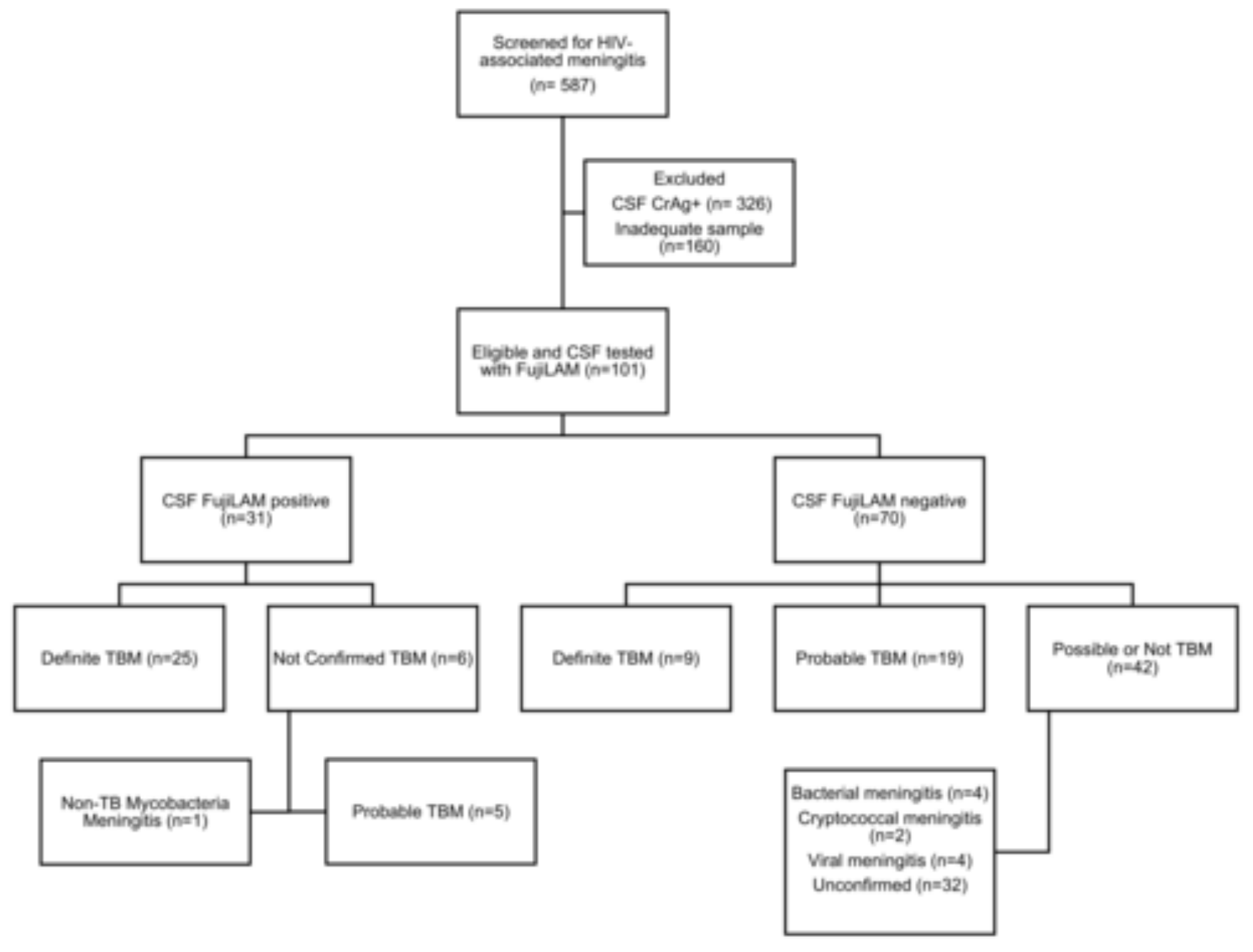


2A

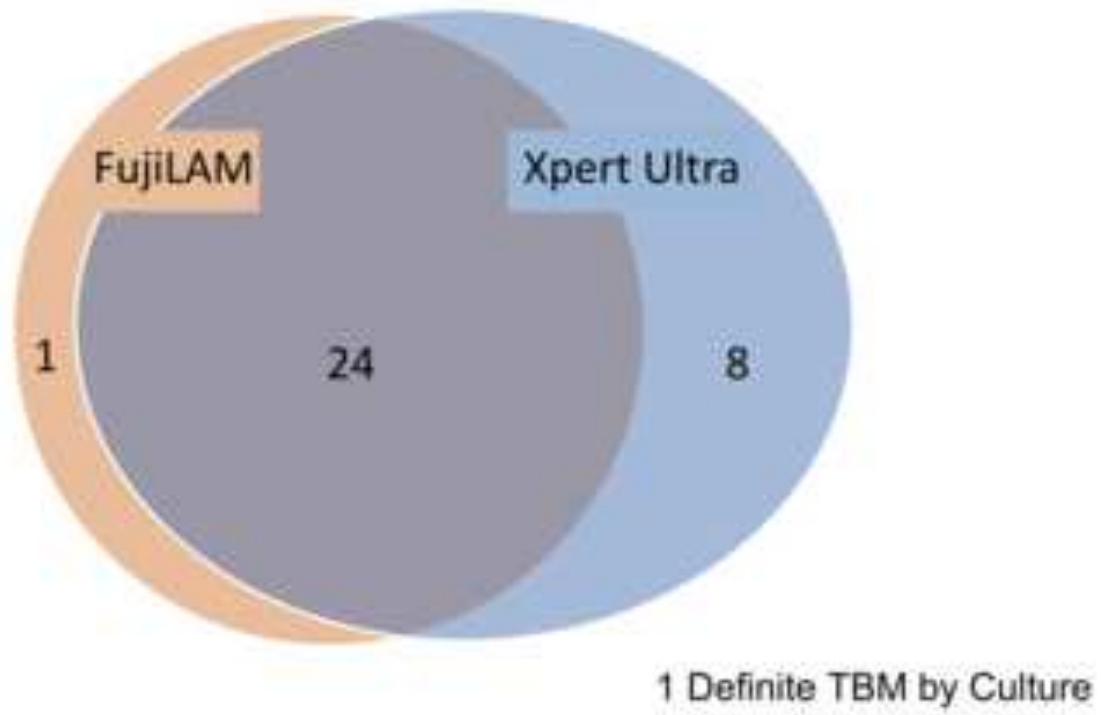

2B

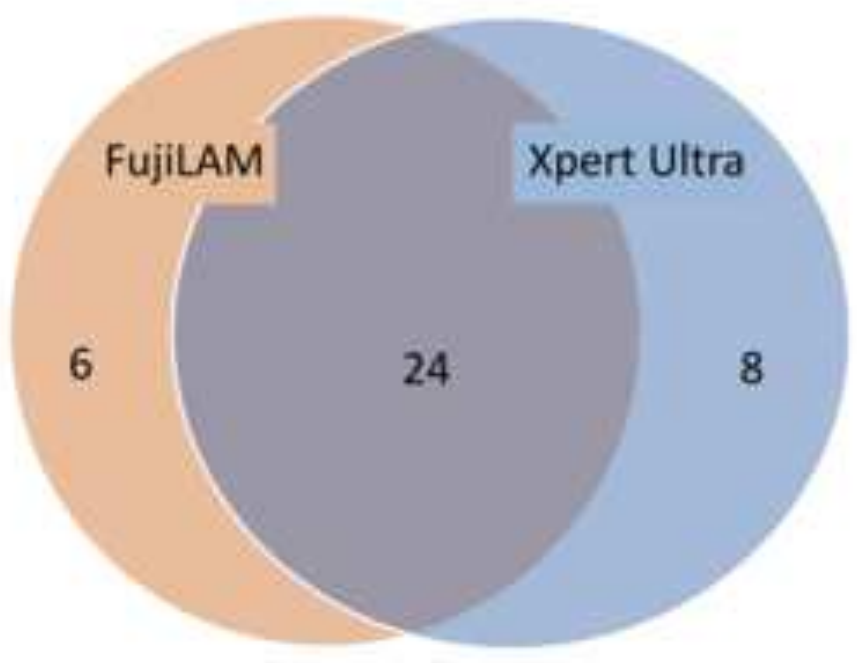

1 Definite TBM by Culture, 19 Probable TBM unconfirmed 


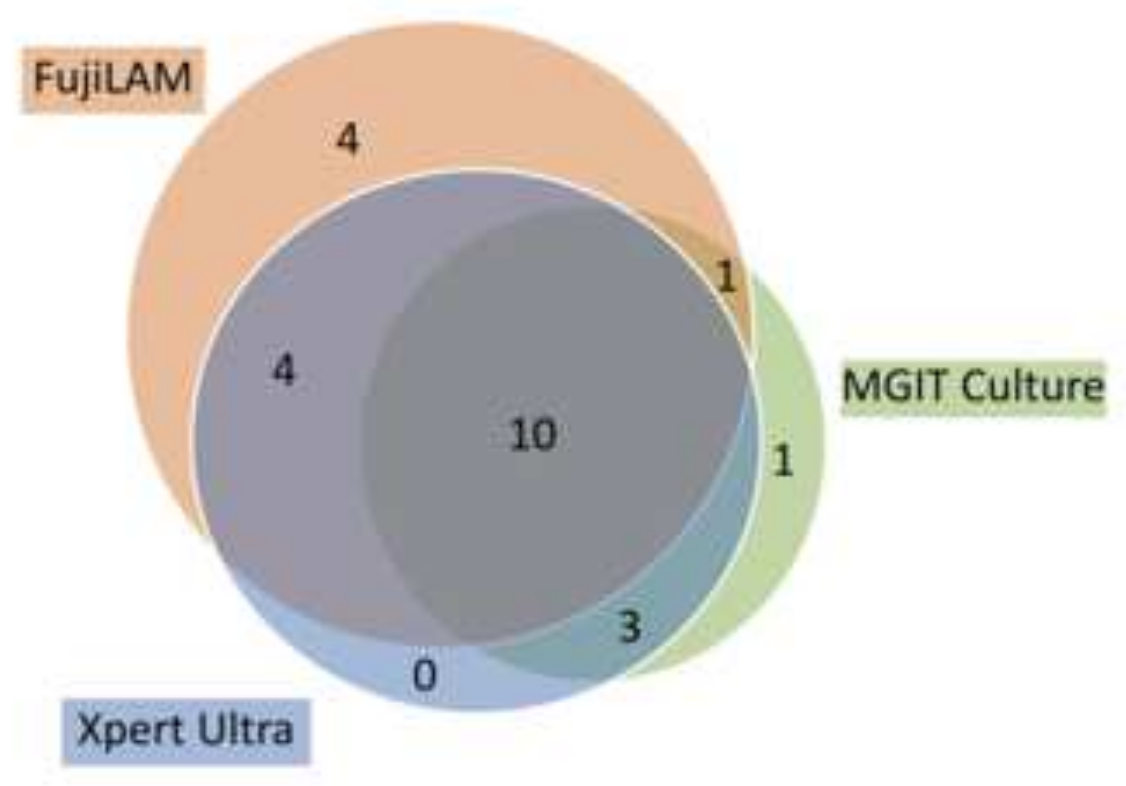

